\title{
Dynamic modelling of axle tramp in a sport type car
}

\author{
Ali Zargartalebi* and Kourosh Heidari Shirazi \\ Mechanical Engineering Department, Faculty of Engineering, Shahid Chamran University, Ahvaz, Iran
}

Received 28 August 2012

Revised 22 January 2013

Accepted 22 January 2013

\begin{abstract}
One of the most significant dynamic aspects of coupled vibration of transmission system and dependent type suspension systems is axle tramp. The tramp is defined as undesirable oscillation of rigid live axle around roll axis. In spite of utilizing powerful engines in some type of sport cars, tramp occurrence causes loss of longitudinal performance. The aim of this paper is to derive a mathematical model for predicting and classifying of the tramp. A parameter study reveals that, some parameters such as engine torque, moving parts moment of inertia, car and wheels weight and the material used in suspension system play important role in controlling the tramp. It is shown that large difference between sprung and unsprung mass moment of inertia around the roll-axis, low vehicle mass, short rear track and medium damping values have significant effects on the severity of tramp.
\end{abstract}

Keywords: Axle tramp, vehicle dynamic, axle vibration, sport car

\section{Introduction}

Traction force in driving wheels is one of the crucial factors in a car longitudinal acceleration performance. The traction and braking forces depend on some parameters such as vertical reaction force on contact patch, side slip angle, camber, longitudinal slip percentage, tyre-road friction coefficient and driving condition. Among the parameters, vertical load on contact patch has more importance in producing enough traction. However, when the tyre separating the road e.g., due to hoping motion occurrence, the vertical force reach down to zero and the tyre traction is lost. According to SAE J670e - SAE Vehicle Dynamics Terminology [11], hop is the vertical oscillatory motion of a wheel between the road surface and the sprung mass. Axle tramp occurs when the right and left side wheels hoping out of phase [11]. Tramp is an undesirable nonlinear vibration of the live axle in a car which is affected by suspension's vertical stiffness, mass of components and internal frictions. From the empirical observations, professional sport car tuners know that the lower mass of components, the higher power-torque engine, the more compliant suspension (high ride comfort) in the transmission system and the heavier axle are some important reason for exciting the axle to oscillate. Increase of the tramp amplitude leads to decrease of wheel's vertical reaction force, loss of longitudinal slip, severe vibrations of sprung mass and consequently, reducing the longitudinal acceleration, inducing skate motion, and more discomfort [12].

Sharp [10] in 1969 investigated tramp under braking condition and used an 11 degrees of freedom model including axle shaft flexural rotation, longitudinal displacement of engine gearbox and also symmetric (longitudinal and vertical) and asymmetric (roll, yaw and pitch) axle motions. Sharp considered the longitudinal axle mounting

\footnotetext{
* Corresponding author: Ali Zargartalebi, Mechanical Engineering Department, Faculty of Engineering, Shahid Chamran University, Ahvaz, PO Box: 135, Iran. Tel.: +98916 312 6783; E-mail: a.zargartalebi@yahoo.com.
} 


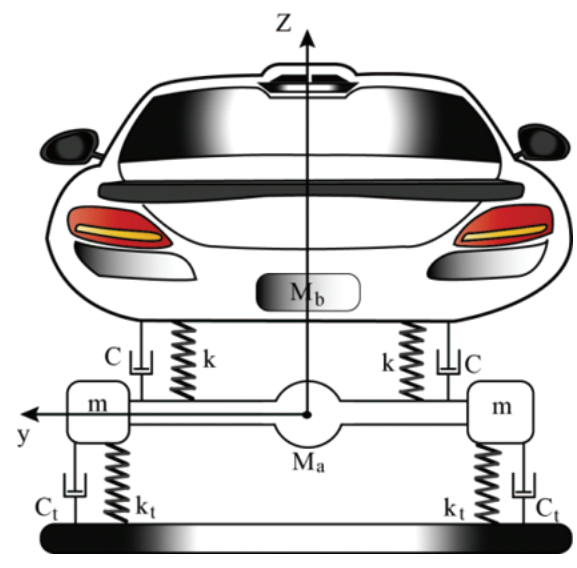

Fig. 1. Schematic view of systems studied.

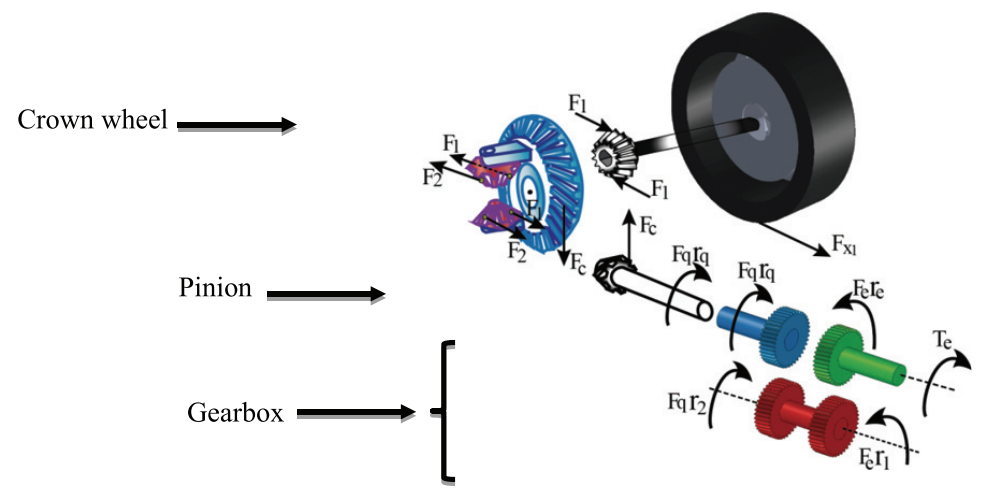

Fig. 2. Schematic view of transmission system.

stiffness as an important parameter for stability of the system and concluded that minimum stability occurs for the symmetric mode when the axle longitudinal translation and bounce natural frequencies are equal and in asymmetric mode while the axle yaw and roll natural frequencies are equal. Sharp and Jones [8] also developed a mathematical model of truck tandem axle suspension and transmission system in which braking and generation of longitudinal tyre-road forces were considered. Sharp [9] considered a simpler situation for tramp phenomenon under braking condition which includes longitudinal, vertical and pitch degrees of freedom. Simple modelling and simulation were advantages of this model and minimum stability yields when longitudinal stiffness becomes a little higher than the tyre vertical stiffness. In addition to the previous studies, in recent years, there has been an increasing interest in problems caused by tramp. Kramer [5] has linked axle tramp to the occurrence of the vehicle skate. There have been also some researches on the effect of delaminated tyre after a tread separation on the handling [1,4].

Beside the previous works, this study is aimed at focusing on more logical modelling. This is provided with employing a realistic engine model, a more complete model of gear train and transmission system, and an experimental tyre model based Pacejka modelling method (Pacejka 2002). In Section 2 based on the specifications of the sport cars a rear wheel drive (RWD) vehicle is selected to establish a dynamic model. The governing equations of motion are derived and converted to dimensionless form. In Section 3 the dimensionless equations of motion are numerically solved. In Section 4 the important parameters on tramp occurrence are determined and role of each parameter in intensity of tramp as well as vibration characteristics such as axle tramping time (ATT), stability types and regions are investigated. In Section 5 concluding remarks are presented.

\section{System model}

Figure 1 shows a schematic model of a RWD rigid axle vehicle. The axle is connected to the vehicle frame by the suspension system springs and dampers. The axle and the frame can freely move vertically and rotate around roll axis and the two rear tyres can rotate independently. Therefore, the model possesses six degrees of freedom. The engine behavior is modeled based on the calculation of the generated engine torque, which is divided into four torques including combustion, mass, friction and load torques $[3,6,7]$ however the friction torque is assumed to be zero. The detail of modelling can be seen in Appendix (A). The vertical dynamic behavior of each tyre is modeled by a set of nonlinear spring and damper. Longitudinal tyre dynamic is followed from Pacejka (2002) model [2] Appendix (B). The vehicle consists of the sprung and the unsprung masses. The tyres masses are included in the unsprung mass. The longitudinal dynamics of the vehicle is neglected and the road surface is assumed to be smooth.

\section{Equations of motion}

Equations of motion include three types of equations namely equilibrium (kinetic relations), compatibility (kinematic relations) and constitutional (the materials and the elements physical behaviors). In the modeling the sub- 
systems including, the engine, the gear train, the differential, the wheels, the axle shafts and the vehicle frame are considered. Free body diagrams of transmission system components are depicted in Fig. 2. Based on the diagrams, the equations of motion for the engine output shaft and the gearbox are presented through Eqs (1) to (3).

$$
\begin{aligned}
& T_{e}-F_{e} r_{e}=\ddot{\phi}_{g} I_{g} \\
& F_{e} r_{1}=F_{q} r_{2} \\
& \dot{\phi}_{g}=N_{t_{i}} \dot{\phi}_{p} \rightarrow i=1,2, \ldots
\end{aligned}
$$

where $I_{g}$ and $T_{e}$ in Eq. (1) represent, moment of inertia and output torque of the engine, $F_{e}$ and $F_{q}$ in Eq. (2) show the forces exerted on input and output shafts of the gearbox, and $\dot{\phi}_{g}$ and $\dot{\phi}_{p}$ in Eq. (3) represent angular velocity of input and output shafts of the gearbox, respectively. The equations of motion of the drive shaft (pinion), the differential and the wheels are as follows:

$$
\begin{aligned}
& -F_{q} r_{q}+F_{c} r_{p}=\ddot{\phi}_{p} I_{p} \\
& F_{c} r_{c}-\left(2 F_{1} r_{a}+2 F_{2} r_{a}\right)=\ddot{\phi}_{c} I_{c} \\
& \left\{\begin{array}{l}
F_{1} r_{h}+F_{2} r_{h}=\ddot{\phi}_{h} I_{h} \\
I_{h} \approx 0
\end{array} \quad \Rightarrow F_{1} \approx F_{2}\right. \\
& 2 F_{1} r_{a}-F_{x_{1}} R=\ddot{\phi}_{1} I_{1} \\
& 2 F_{2} r_{a}-F_{x_{2}} R=\ddot{\phi}_{2} I_{2}
\end{aligned}
$$

where, $I_{p}$ and $r_{p}$ in Eq. (4) are moment of inertia and equivalent radius of pinion, respectively. The reactive forces generated in planetary gear of the differential is denoted by $F_{1}$ and $F_{2}$, and equivalent radius of planetary gear by $r_{a}$ and moment of inertia of the crown wheel by $I_{c}$ in Eq. (5). In derivation of Eq. (6) it is assumed that the planetary gears moment of inertia is negligible. The traction forces on the wheels are denoted by $F_{x_{1}}, F_{x_{2}}$ in Eqs (7) and (8). The effective radius of wheel and moment of inertia around $y$ axis are denoted by $R, I_{1}$ and $I_{2}$, respectively. From kinematic relations between angular velocity of wheel $\left(\dot{\phi}_{1}, \dot{\phi}_{2}\right)$, crown wheel $\left(\dot{\phi}_{c}\right)$ and pinion $\left(\dot{\phi}_{p}\right)$ the compatibility equations can be expressed as follows:

$$
\begin{aligned}
& \dot{\phi}_{c}=\left(r_{p} / r_{c}\right) \dot{\phi}_{p} \\
& \dot{\phi}_{c}=\left(\dot{\phi}_{1}+\dot{\phi}_{2}\right) / 2
\end{aligned}
$$

To derive equations of motion for vertical translation and rolling motion of the axle as well as the vehicle frame the Lagrangian mechanics approach is employed. The detail of calculation of kinetic and potential energies can be found in Appendix (C). The equations of motion are as follows:

$$
\begin{aligned}
& (M+2 m) \ddot{z}-2 C\left(\dot{z}_{b}-\dot{z}\right)+2 C_{t} \dot{z}-4 k\left(z_{b}-z\right)+2 k_{t 1} z+k_{t 2}\left\{(z+s \phi)^{3}+(z-s \phi)^{3}\right\}=Q_{z} \\
& M_{b} \ddot{z}_{b}+2 C\left(\dot{z}_{b}-\dot{z}\right)+4 k\left(z_{b}-z\right)=0 \\
& \left(I_{x}+2 i_{x}+2 m s^{2}\right) \ddot{\phi}+2 C l^{2}\left(\dot{\phi}-\dot{\phi}_{b}\right)+4 k l^{2}\left(\phi-\phi_{b}\right)+2 s^{2} k_{t 1} \phi+k_{t 2} s\left\{(z+s \phi)^{3}+(z-s \phi)^{3}\right\} \\
& \quad+2 s^{2} C_{t} \dot{\phi}=Q_{\phi} \\
& I_{x b} \ddot{\phi}_{b}+2 C l^{2}\left(\dot{\phi}_{b}-\dot{\phi}\right)+4 k l^{2}\left(\phi_{b}-\phi\right)=Q_{\phi b}
\end{aligned}
$$

Equations (11) and (12) describe the vertical translational motion of the axle and the vehicle frame and Eqs (13) and (14) describe the rotational dynamics around the roll axis. $Q_{i}$ 's denote the associated generalized forces and $k$ and $C$ are stiffness and damper coefficients of suspension system, respectively. The wheels are considered as nonlinear hardening springs with linear and non-linear parts stiffness coefficients as, $k_{t 1}$ and $k_{t 2}$, the parameters, $M, M_{b}$ and $m$ denote the masses of the axle, the vehicle frame and the wheels, respectively. The moment of inertia of the axle, the vehicle frame and the wheels are denoted by $I_{x}, I_{x b}$ and $i_{x}$, respectively. The generalized forces $Q_{i}$ 's are given as follows:

$$
Q_{z}=F_{Z_{1}}+F_{Z_{2}}
$$


Table 1

System parameters and their values as used in the modeling

\begin{tabular}{|c|c|c|c|}
\hline Symbol & Parameter & Value & Unit \\
\hline$s$ & Axle length & 0.8 & $\mathrm{~m}$ \\
\hline$l$ & Spring distance & 0.65 & $\mathrm{~m}$ \\
\hline$C$ & Suspension damping coefficient & 1200 & $\frac{N . s}{m}$ \\
\hline$C_{t}$ & Vertical tire damping coefficient & 100 & $\frac{\mathrm{N} \cdot \mathrm{s}}{\mathrm{m}}$ \\
\hline$F_{c}$ & Force between pinion and crown wheel & - & $\stackrel{\mathrm{m}}{\mathrm{N}}$ \\
\hline$F_{e}$ & Gearbox input force & - & $\mathrm{N}$ \\
\hline$F_{1}, F_{2}$ & Sun gears forces in differential & - & $\mathrm{N}$ \\
\hline$F_{q}$ & Gearbox output force & - & $\mathrm{N}$ \\
\hline$F_{x 1}, F_{x 2}$ & Longitudinal tire to road forces (1:left, 2 :right) & - & $\mathrm{N}$ \\
\hline$F_{Z_{1}}, F_{Z_{2}}$ & Vertical tire forces (1:left, 2:right) & - & $\mathrm{N}$ \\
\hline$g$ & Acceleration due to gravity & 9.81 & $\frac{m}{2}$ \\
\hline$I_{c}$ & Crown wheel inertia & 0.02 & kg.m $\mathrm{m}^{2}$ \\
\hline$I_{g}$ & Inertia of gearbox input & 0.06 & kg.m ${ }^{2}$ \\
\hline$I_{h}$ & Planet gear inertia & - & kg. $\mathrm{m}^{2}$ \\
\hline$I_{p}$ & Pinion and propeller shaft inertia & 2 & $\mathrm{~kg} \cdot \mathrm{m}^{2}$ \\
\hline$I_{x}$ & Roll inertia of axle & 30 & $\mathrm{~kg} . \mathrm{m}^{2}$ \\
\hline$I_{x b}$ & Roll inertia of body frame & 840 & kg.m ${ }^{2}$ \\
\hline$i_{x}$ & Camber inertia of one tire about axes through mass center & 0.9 & kg.m ${ }^{2}$ \\
\hline$I_{1}, I_{2}$ & Polar moment of inertia of one wheel about axes through mass center (1:left, 2 :right) & 1.2 & kg. $\mathrm{m}^{2}$ \\
\hline$J$ & Crankshaft inertia & 0.55 & $\mathrm{~kg} \cdot \mathrm{m}^{2} \mathrm{r} \cdot \mathrm{c} \cdot \mathrm{c}$ \\
\hline$K$ & Suspension stiffness coefficient & $4 \times 10^{4}$ & $\frac{N}{m}$ \\
\hline$K_{t 1}$ & Vertical tire stiffness linear coefficient & $2.5 \times 10^{5}$ & $\frac{m}{m}$ \\
\hline$K_{t 2}$ & Vertical tire stiffness nonlinear coefficient & $7.5 \times 10^{9}$ & $\frac{\frac{m}{N}}{m^{3}}$ \\
\hline$M$ & Mass of axle & 60 & $\mathrm{~kg}$ \\
\hline$M_{b}$ & Mass of body frame on rear tires & 450 & $\mathrm{~kg}$ \\
\hline$m$ & Mass of one wheel & 8 & $\mathrm{~kg}$ \\
\hline$N_{t_{i}}$ & Gear status & 1 & - \\
\hline$\dot{\phi}_{1}$ & Left wheel angular velocity & - & $\frac{\mathrm{rad}}{\mathrm{s}}$ \\
\hline$\dot{\phi}_{2}$ & Right wheel angular velocity & - & $\frac{\mathrm{rad}}{\mathrm{s}}$ \\
\hline$\dot{\phi}_{c}$ & Crown wheel angular velocity & - & $\frac{\mathrm{rad}}{\mathrm{r}}$ \\
\hline$\dot{\phi}_{g}$ & Gearbox input angular velocity & - & $\frac{\mathrm{rad}}{s}$ \\
\hline$\dot{\phi}_{h}^{9}$ & Planet gear angular velocity & - & $\underline{\mathrm{rad}}$ \\
\hline$\dot{\phi}_{p}$ & Pinion angular velocity & - & $\frac{\mathrm{rad}}{\mathrm{rad}}$ \\
\hline$Q_{i}$ & Generalized forces & _- & $\stackrel{\mathrm{s}}{-}$ \\
\hline$R$ & Wheel radius & 0.31 & $\mathrm{~m}$ \\
\hline$r_{a}$ & Sun Gear radius in differential & 0.05 & $\mathrm{~m}$ \\
\hline$r_{c}$ & Crown wheel radius in differential & 0.1 & $\mathrm{~m}$ \\
\hline$r_{e}$ & Gear radius at gearbox input & 0.045 & $\mathrm{~m}$ \\
\hline$r_{h}$ & Planet gear radius & 0.05 & $\mathrm{~m}$ \\
\hline$r_{p}$ & Pinion radius & 0.03 & $\mathrm{~m}$ \\
\hline$r_{1}$ & Gear radius in gearbox & 0.09 & $\mathrm{~m}$ \\
\hline$r_{2}$ & Gear radius in gearbox & 0.05 & $\mathrm{~m}$ \\
\hline$r_{q}$ & Gear radius at gearbox output & 0.1 & $\mathrm{~m}$ \\
\hline$T_{e}$ & Engine torque & - & N.m \\
\hline$Z$ & Vertical displacement of axle mass center & - & $\mathrm{m}$ \\
\hline$Z_{b}$ & Vertical displacement of body frame & - & $\mathrm{m}$ \\
\hline$\Delta$ & Tire equilibrium compression & - & $\mathrm{m}$ \\
\hline$\phi$ & Roll displacement of axle & - & $\mathrm{rad}$ \\
\hline$\phi_{b}$ & Roll displacement of body frame & - & $\mathrm{rad}$ \\
\hline$\theta$ & Crank angle & - & $\mathrm{rad}$ \\
\hline
\end{tabular}

where:

$$
\begin{aligned}
& F_{Z_{1}}=k_{t 1}(z+s \phi-\Delta)+k_{t 2}(z+s \phi-\Delta)^{3}+C_{t}(\dot{z}+s \dot{\phi}) \\
& F_{Z_{2}}=k_{t 1}(z-s \phi-\Delta)+k_{t 2}(z-s \phi-\Delta)^{3}+C_{t}(\dot{z}-s \dot{\phi}) \\
& Q_{\phi}=s\left(F_{Z_{1}}-F_{Z_{2}}\right)-F_{q} r_{q}
\end{aligned}
$$



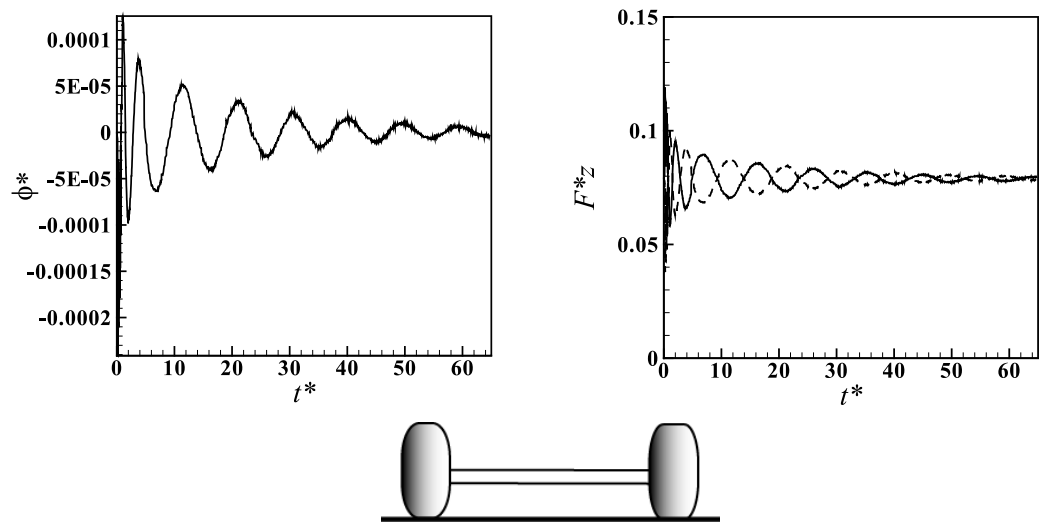

(a)
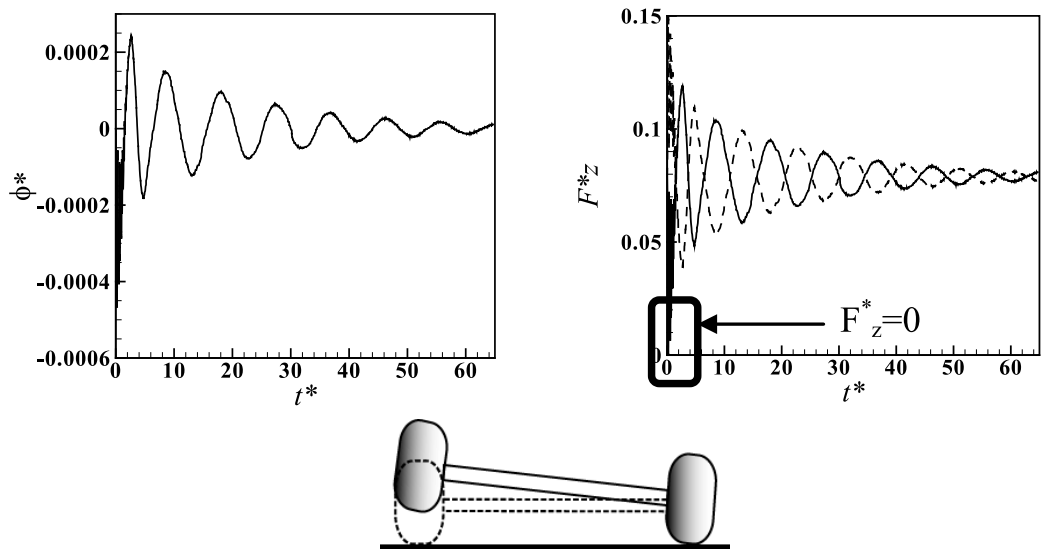

(b)

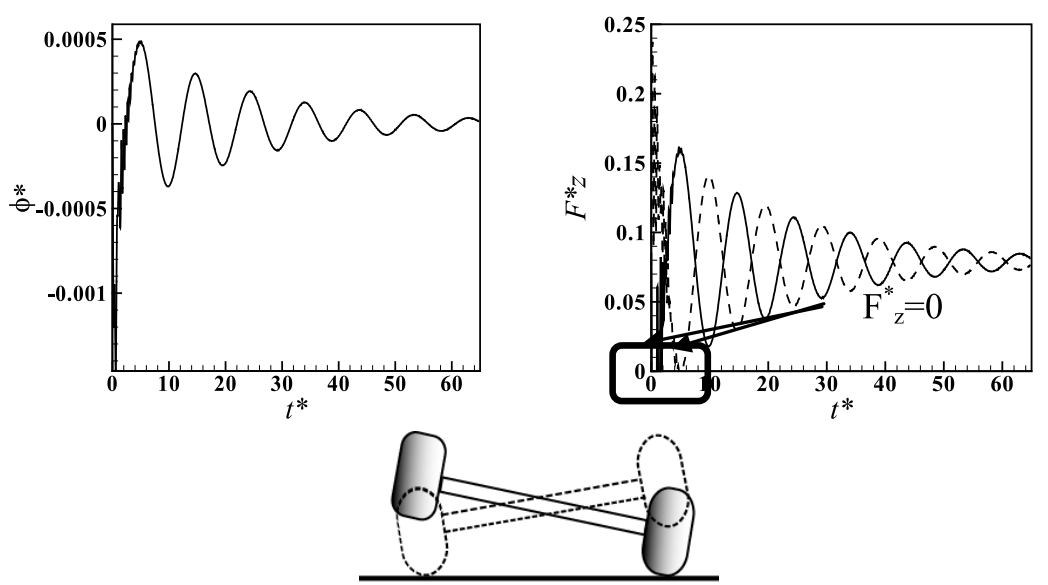

(c)

Fig. 3. The stability statuses (a) Sub-critical (b) Trans-critical (c) Critical.

$$
Q_{\phi b}=F_{q} r_{q}
$$

The vertical tyre forces in Eq. (15) are restricted to positive values. Realistic parameters for the system are summarized in Table 1. The procedure of changing the equations to the dimensionless form is presented in the Appendix (D). 


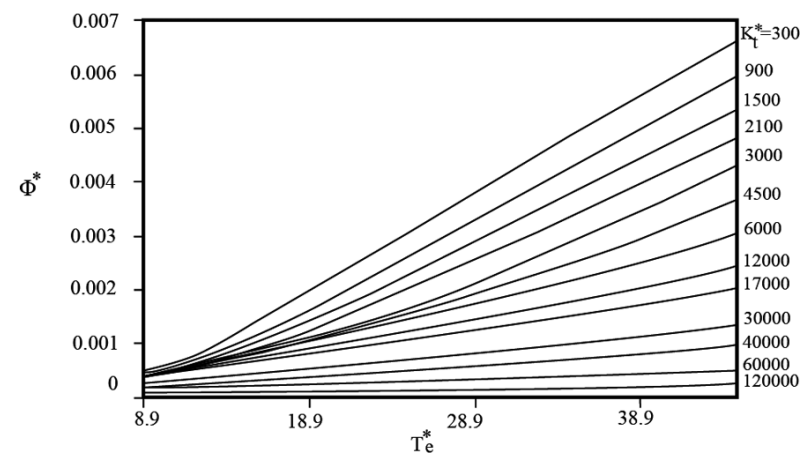

Fig. 4. Effect of $K_{t}^{*}$ and $T_{e}^{*}$ on dimensionless vibration amplitude of axle.

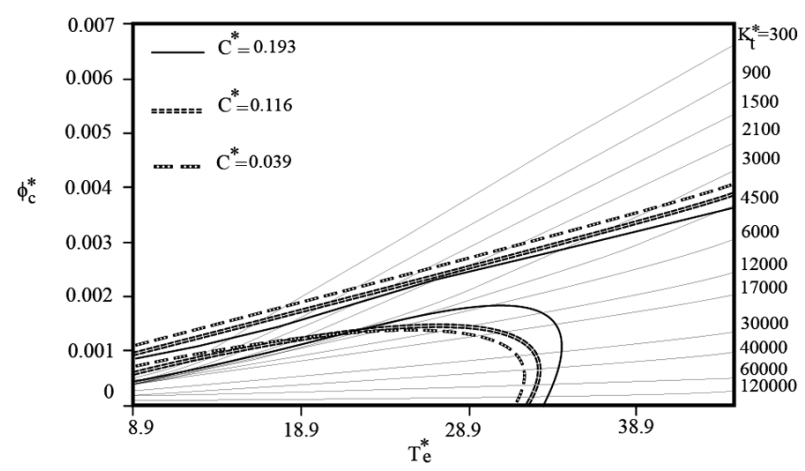

Fig. 6. The regions movement according to $C^{*}$ variations.

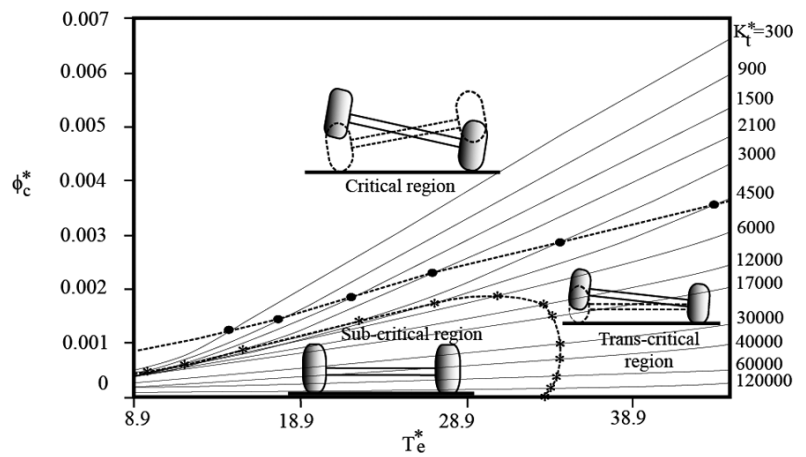

Fig. 5. The stability regions for various $K_{t}^{*}$ and $T_{e}^{*}\left(C^{*}=0.193\right)$.

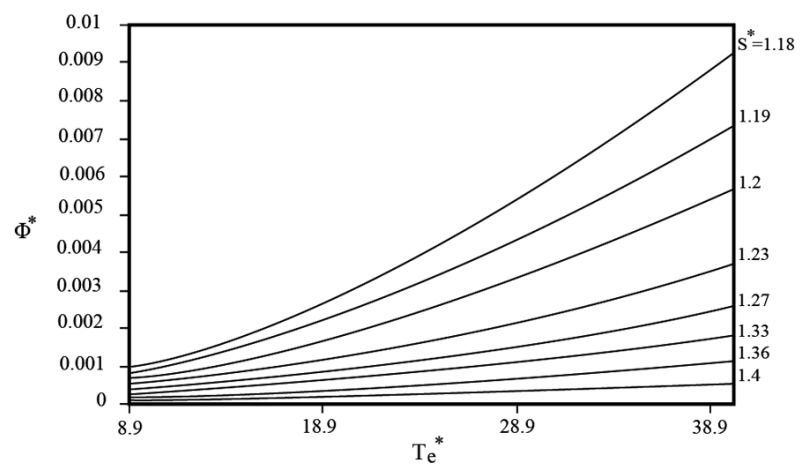

Fig. 7. Effect of $S^{*}$ and $T_{e}^{*}$ on dimensionless vibration amplitude of axle $\left(C^{*}=0.116\right)$.

\section{Results and discussions}

The dimensionless equations are used to simulate the whole model dynamic response to the input excitations for predicting the axle tramp occurrence conditions and classification. The system is subjected to the input engine torque and tyre-road forces as well.

The first investigation is organized to study the effect of engine torque on vibration amplitude of the axle. To this end, dimensionless vibration amplitude of axle $\Phi^{*}$ versus dimensionless engine torque $T_{e}^{*}=T_{e} \tau^{2} / I_{g}$ is plotted. According to Fig. 3, three types of rotational stability of the axle can be recognized. In type (a) the subcritical case, the axle oscillates without wheel hoping. In type (b), the trans-critical case, one wheel hop occurs and one wheel keeps its contact to the ground. In the hoping wheel dimensionless force $\left(F_{z}^{*}=F_{z} /(k s)\right)$ comes to zero. In type (c), the critical case, both wheels of the axle show the hoping motion repeatedly. In Fig. 4 a family of curves which shows the axle vibration amplitude versus $T_{e}^{*}$ are generated. Each curve has certain value of vertical dimensionless wheel stiffness $\left(K_{t}^{*}=K_{t 2} / K_{t 1}\right)$. The effect of $K_{t}^{*}$ on shape of each curve is obvious. It is seen that larger $K_{t}^{*}$, e.g., due to more tyre pneumatic pressure causes less sensitivity of the amplitude changing to $T_{e}^{*}$.

\subsection{Stability regions of the axle tramp}

The three stability regions of the rotational axle vibration are depicted in Fig. 5. In this figure the effect of two parameters $T_{e}^{*}$ and $K_{t}^{*}$ on appearance and developing of the three region of axle tramp is shown. In order to define the boundary between three regions, the axle angle is replaced by critical axle roll angle $\phi_{c}^{*}$. One can say that for enough small $K_{t}^{*}$ the critical case occurs even in small $T_{e}^{*}$ and for enough large $T_{e}^{*}$ the trans-critical case occurs regardless to $K_{t}^{*}$. On the other hand for $K_{t}^{*} \geqslant 4.5 \times 10^{4}$ and for $T_{e}^{*} \leqslant 34$ the axle vibrating behavior located into the subcritical region. Effect of dimensionless suspension damping $\left(C^{*}=C /\left(2 \sqrt{k M_{b}}\right)\right)$ on boundary curves of 


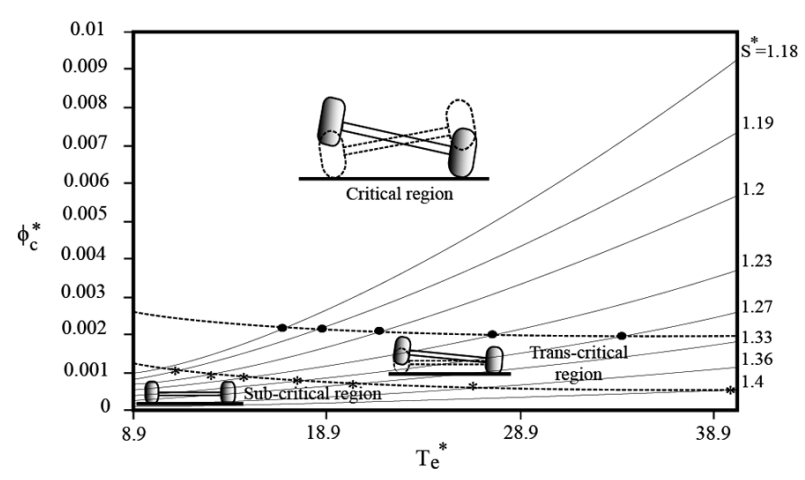

Fig. 8. The stability regions for various $S^{*}$ and $T_{e}^{*}\left(C^{*}=0.116\right)$.

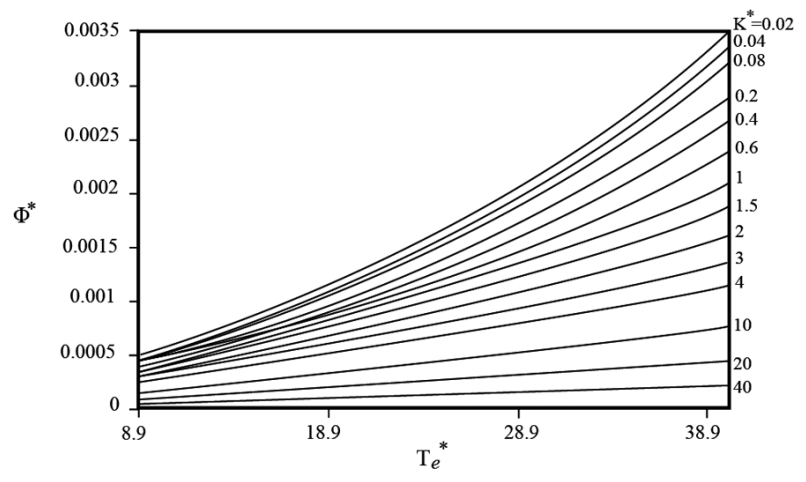

Fig. 9. Effect of $K^{*}$ and $T_{e}^{*}$ on dimensionless vibration amplitude of axle $\left(C^{*}=0.116\right)$.

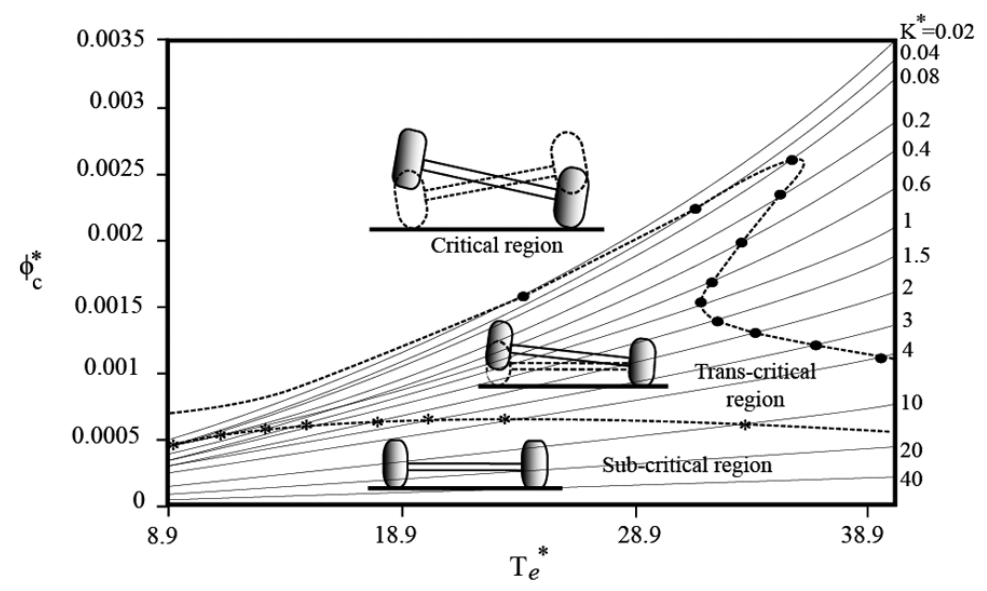

Fig. 10. The stability regions for various $K^{*}$ and $T_{e}^{*}\left(C^{*}=0.116\right)$.

the stability regions can be seen in Fig. 6. Referring to Fig. 6 one can see the two opposite effects from $C^{*}$ on the stability boundary movement. Increase of $C^{*}$ when $T_{e}^{*}$ is greater than 23.3, extends the subcritical region, and when $T_{e}^{*}$ is small, contracts the subcritical region. Also increase of $C^{*}$ improves the trans-critical region. Dependency of the axle vibration amplitude to dimensionless axle length $S^{*}=s / l$ is another parameter study. Figures 7 and 8 show the dimensionless amplitude of the axle vibration and stability regions, when $S^{*}$ get different values. According to both the figures, larger values of $S^{*}$ causes more stable axle vibration and suppressing the trans-critical and critical regions. Regarding to demonstrate the effect of relative elasticity of suspension system and tyre, on the axle vibration the dimensionless suspension stiffness $K^{*}=K / K_{t 1}$ is considered as parameter. The dimensionless axle vibration amplitude $\Phi^{*}$ and the stability regions for different values of $K^{*}$ are plotter in Figs 9 and 10, respectively. One can see that $K^{*}$ could be another important parameter on the axle tramp. Larger values of $K^{*}$ suppress the trans-critical and critical regions. However, $K^{*}$ has significant effect on the vehicle handling as well as ride comfort performance. Larger $K^{*}$ causes better handling and worse the ride. In other words increase of $K^{*}$ enhances the handling and suppresses the axle tramp.

\subsection{The axle tramping time}

The axle tramp is a transient behavior such that after a period of time it is disappeared. The axle tramp timing (ATT) is defined in a dimensionless form by dividing by a reference time. Some system parameters affect the ATT. Graph of the ATT versus $K^{*}$ and moment of inertia dimensionless parameter $\left(I^{*}=I_{x} / I_{x b}\right)$ are demonstrated in Fig. 11. One can see in Fig. 11 that, the ATT is slightly reduced at a small initial zone (about $0.02 \leqslant K^{*} \leqslant 0.04$ ). 


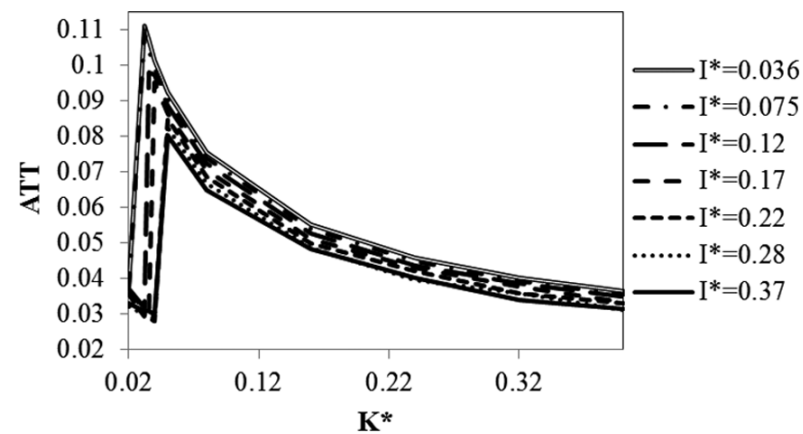

Fig. 11. The critical tramping time for $K^{*}$ and $I^{*}$ variations $\left(T_{e}^{*}=\right.$ 26.5).

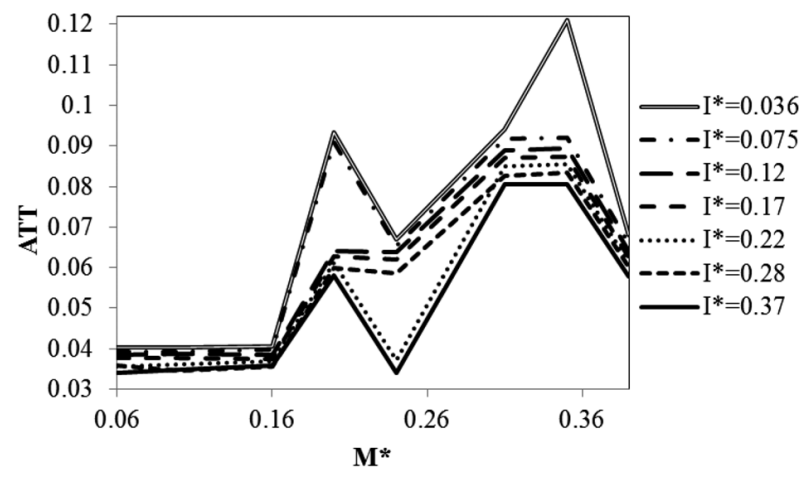

Fig. 13. The critical tramping time for $M^{*}$ and $I^{*}$ variations $\left(T_{e}^{*}=\right.$ 26.5).

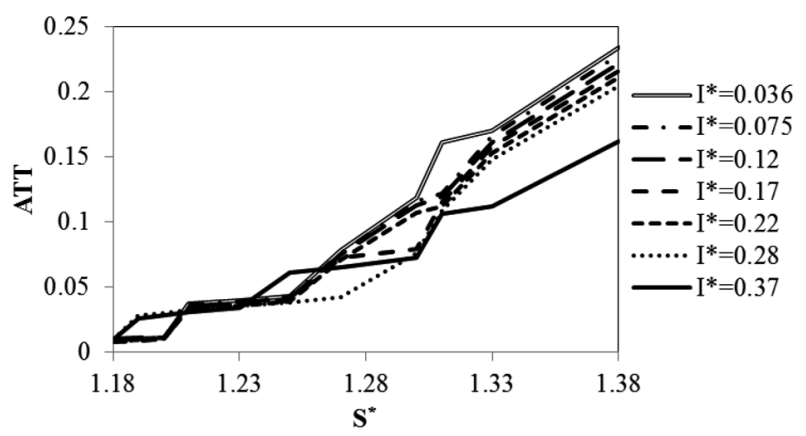

Fig. 12. The critical tramping time for $S^{*}$ and $I^{*}$ variations $\left(T_{e}^{*}=\right.$ 26.5).

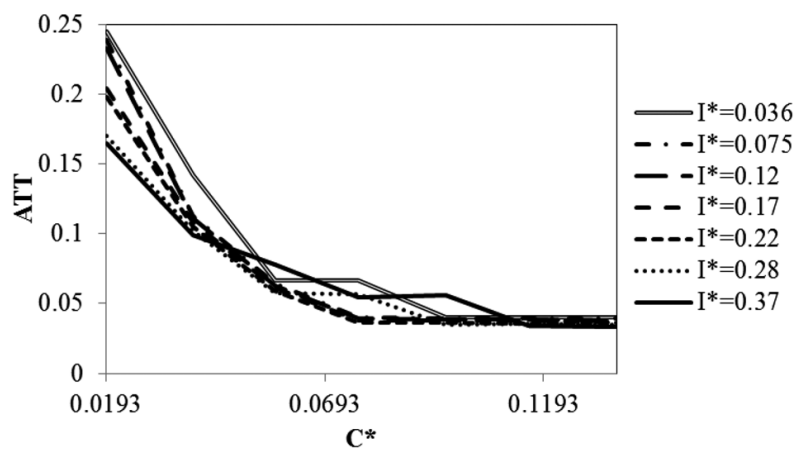

Fig. 14. The critical tramping time for $C^{*}$ and $I^{*}$ variations $\left(T_{e}^{*}=\right.$ 26.5).

However this behavior is followed by a suddenly increase of the ATT and appearance of a peak value (about $0.04 \leqslant$ $K^{*} \leqslant 0.06$ ). For enough large values of dimensionless chassis stiffness (about $K^{*} \geqslant 0.12$ ) the ATT asymptotically decreased. The dimensionless moment of inertia $I^{*}$ plays role of a controlling parameter for the ATT. The increase of $I^{*}$, the decrease the peak value and shifting the whole curve down. As shown in Fig. 12 the increase of dimensionless axle length $\left(S^{*}\right)$, the increase of ATT. The ATT tends to higher values for larger $S^{*}$. This means that the larger ratio of axle length to the spring distance makes longer tramp.

The dimensionless mass $M^{*}=M / M_{b}$ is another parameter to be considered in study of the ATT. Roughly speaking, the family graphs in Fig. 13, shows that there is no general conclusion about the effect of $M^{*}$ on the ATT. However partially and in some ranges of $M^{*}$ changing of the ATT for different values of $I^{*}$ follows the obvious increasing-decreasing patterns.

Nevertheless, according to Fig. 14 the significant role of the dimensionless damping $C^{*}$ on the ATT is seen. The increase of $C^{*}$, the decrease of the ATT for all values of $I^{*}$. Another conclusion obtained from Fig. 14 is asymptotic behavior of the ATT versus $C^{*}$. The controlling effect of $C^{*}$ in limiting the ATT gradually decreased for larger value of $C^{*}$. This means that there is a minimum value for $C^{*}$ to obtain the most effect on the tramp. According to Fig. 14 $C^{*} \geqslant 0.1$ can be a good suggestion.

\section{Conclusions}

In this research the axle tramp behavior for a sport type car has been considered. A dynamic model has been built for obtaining different dynamical behavior the considered system. The model contains effective parameters $\left(T_{e}^{*}, K_{t}^{*}\right.$, $K^{*}, I^{*}, S^{*}, C^{*}$ ) of the system and the dynamic simulation reveals the effect of each parameter on characteristics $\left(\phi_{c}^{*}\right.$, stability regions and the ATT) of the axle tramp. The following conclusions can be summarized form the results: 
- The high the engine torque, the increase the tramp critical region.

- The higher ratios of the sprung to unsprung mass moments of inertia, the intensifying the axle tramp.

- The higher ratios of the axle length to the spring distance, the intensifying the tramp and longer ATT.

- There is an effective value for dimensionless suspension damping $C^{*} \geqslant 0.1$ by which the tramp significantly is controlled.

- The more ratio of the spring distance to the tread $\left(S^{*}\right)$ in each axle, the more limiting the tramp.

- The dimensionless mass $\left(M^{*}\right)$ has opposite and different effects on tramp and no general rule can be seen.

\section{Appendix A: Engine modelling}

The total torque is approximated by considering four basic torques:

- Combustion Torque ( $\left.T_{\mathrm{comb}}\right)$

- Mass Torque $\left(T_{m}\right)$

- Friction Torque $\left(T_{f}\right)$

- Load Torque ( $\left.T_{\text {load }}\right)$

The crankshaft torque is described using the balancing equation [10]:

$$
J \ddot{\theta}+T_{\text {comb }}-T_{f}-T_{\text {load }}-T_{m}(\theta, \dot{\theta}, \ddot{\theta})=0
$$

where $J$ is the crankshaft inertia and $\theta, \dot{\theta}, \ddot{\theta}$ is the crank angle, angular velocity and angular acceleration respectively. The combustion torque is described as follows [11]:

$$
T_{\mathrm{comb}}=\frac{\eta_{f} \dot{m}_{f c} Q_{L H V}}{\omega_{e}(t)}
$$

The Friction Torque is neglected. The equation of mass torque is as follows [12]:

$$
T_{m}(\ddot{\theta}, \dot{\theta}, \theta)=J(\theta) \ddot{\theta}+\frac{1}{2} \frac{d J(\theta)}{d \theta} \dot{\theta}^{2}
$$

The Load Torque is the load acting at the crankshaft. Another notation for the load torque is Brake Mean Effective Pressure:

$$
\mathrm{BMEP}=1 / V_{d} \oint_{\xi} T_{l} d \theta
$$

Where $\xi$ represents a cycle. Thus, for each cycle BMEP is:

$$
\mathrm{BMEP}=4 \pi \bar{T}_{l} / V_{d}
$$

\section{Appendix B: Tyre modelling (Pacejka 2002)}

According to the Pacejka 2002 model, for the tyre rolling on a straight line with no slip angle condition we have [9]:

$$
\begin{aligned}
& F_{x}=F_{x 0}\left(\gamma, k, F_{z}\right) \\
& F_{x 0}=D_{x} \sin \left[C_{x} \operatorname{atan}\left\{B_{x} k_{x}-E_{x}\left(B_{x} k_{x}-\operatorname{atan}\left(B_{x} k_{x}\right)\right)\right\}\right]+s_{v_{x}} \\
& k_{x}=\kappa+s_{H_{x}}
\end{aligned}
$$


Table 1.B

Longitudinal force coefficients at pure slip

\begin{tabular}{lcl}
\hline Name & Name used in tyre property file & Explanation \\
\hline$p_{C x 1}$ & PCX1 & Shape factor $C_{f x}$ for longitudinal force \\
$p_{D x 1}$ & PDX1 & Longitudinal friction Mux at $F_{\text {znom }}$ \\
$p_{D x 2}$ & PDX2 & Variation of friction Mux with load \\
$p_{D x 3}$ & PDX3 & Variation of friction Mux with inclination \\
$p_{E x 1}$ & PEX1 & Longitudinal curvature $E_{f x}$ at $F_{\text {znom }}$ \\
$p_{E x 2}$ & PEX2 & Variation of curvature $E_{f x}$ with load \\
$p_{E x 3}$ & PEX3 & Variation of curvature $E_{f x}$ with load squared \\
$p_{E x 4}$ & PEX4 & Factor in curvature $E_{f x}$ while driving \\
$p_{K x 1}$ & PKX1 & Longitudinal slip stiffness $K_{f x} / F_{z}$ at $F_{\text {znom }}$ \\
$p_{K x 2}$ & PKX2 & Variation of slip stiffness $K_{f x} / F_{z}$ with load \\
$p_{K x 3}$ & PKX3 & Exponent in slip stiffness $K_{f x} / F_{z}$ with load \\
$p_{H x 1}$ & PHX1 & Horizontal shift $S_{h x}$ at $F_{\text {znom }}$ \\
$p_{H x 2}$ & PHX2 & Variation of shift $S_{h x}$ with load \\
$p_{V x 1}$ & PVX1 & Vertical shift $S_{v x} / F_{z}$ at $F_{\text {znom }}$ \\
$p_{V x 2}$ & PVX2 & Variation of shift $S_{v x} / F_{z}$ with load \\
\hline
\end{tabular}

where:

$$
\begin{aligned}
& C_{x}=p_{C_{x 1}} \lambda_{C x} \\
& D_{x}=\mu_{x} F_{z} \\
& \mu_{x}=\left(p_{D_{x 1}}+p_{D_{x 2}} . d F_{z}\right)\left(1-p_{D_{x 3}} \gamma_{x}^{2}\right) \lambda_{\mu x} \\
& \gamma_{x}=\gamma \lambda_{\gamma x} \\
& E_{x}=\left(p_{E_{x 1}}+p_{E_{x 2}} d F_{z}+p_{E_{x 2}} d F_{z}^{2}\right)\left\{1-p_{E_{x 3}} \operatorname{sign}\left(k_{x}\right)\right\} \lambda_{E x}
\end{aligned}
$$

The longitudinal slip stiffness:

$$
\begin{aligned}
k_{x} & =F_{z}\left(p_{k_{x 1}}+p_{k_{x 2}} d F_{z}\right) \exp \left(p_{k_{x 3}} d F_{z}\right) \lambda_{k x} \Rightarrow\left(k_{x}=B_{x} C_{x} D_{x}\right) \\
B_{x} & =\frac{k_{x}}{C_{x} D_{x}} \\
S_{H x} & =\left(p_{H_{x 1}}+p_{H_{x 2}} d F_{z}\right) \lambda_{H x} \\
S_{V x} & =F_{z}\left(p_{V_{x 1}}+p_{V_{x 2}} d F_{z}\right) \lambda_{V x} \lambda_{\mu x}
\end{aligned}
$$

Also longitudinal coefficients (Table 1.B) values are as follows:

$$
\begin{aligned}
& \text { PCX1 }=1.839 ; \\
& \text { PDX1 }=1.1387 ; \\
& \text { PDX2 }=-0.11999 ; \\
& \text { PDX3 }=-2.2142 \mathrm{e}-005 ; \\
& \text { PEX1 }=0.62727 ; \\
& \text { PEX2 }=-0.12336 ; \\
& \text { PEX3 }=-0.03448 ; \\
& \text { PEX4 }=-1.5066 \mathrm{e}-005 ; \\
& \text { PKX1 }=18.886 ; \\
& \text { PKX2 }=-3.988 ; \\
& \text { PKX3 }=0.21542 ; \\
& \text { PHX1 }=-0.00033912 ; \\
& \text { PHX2 }=-8.5877 \mathrm{e}-006 ; \\
& \text { PVX1 }=-4.638 \mathrm{e}-006 ; \\
& \text { PVX2 }=1.9874 \mathrm{e}-005 ; \\
& \text { PTX1 }=1.85 ;
\end{aligned}
$$


$\mathrm{PTX} 2=0.000109$

PTX3 = 0.101;

$\gamma=0 ; R_{0}=0.31$

Finally the scaling coefficients values are as follows:

$\mathrm{LFZO}=1 ; \%$ Scale factor of nominal (rated) load $\left(\lambda_{F_{z 0}}\right)$

$\mathrm{LCX}=1 ; \%$ Scale factor of $F_{x}$ shape factor $\left(\lambda_{c x}\right)$

LMUX $=1 ; \%$ Scale factor of $F_{x}$ peak friction coefficient $\left(\lambda_{\mu x}\right)$

$\mathrm{LEX}=1 ; \%$ Scale factor of $F_{x}$ curvature factor $\left(\lambda_{E x}\right)$

LKX $=1 ; \%$ Scale factor of $F_{x}$ slip stiffness $\left(\lambda_{\kappa x}\right)$

LHX $=0 ; \%$ Scale factor of $F_{x}$ horizontal shift $\left(\lambda_{H x}\right)$

$\mathrm{LVX}=0 ; \%$ Scale factor of $F_{x}$ vertical shift $\left(\lambda_{v x}\right)$

LGAX $=1 ; \%$ Scale factor of camber for $F_{x}\left(\lambda_{\gamma x}\right)$

\section{Appendix C: Kinetic and potential energies calculations}

The kinetic and potential energies are calculated as follows:

$$
\begin{aligned}
T= & \frac{1}{2} M \dot{z}^{2}+\frac{1}{2} M_{b} \dot{z}_{b}^{2}+\frac{1}{2} I_{x} \dot{\phi}^{2}+\frac{1}{2} I_{x b} \dot{\phi}_{b}^{2} \\
& +\frac{1}{2} m\left\{(\dot{z}+s \dot{\phi})^{2}+(\dot{z}-s \dot{\phi})^{2}\right\}+i_{y}\left(\left(\dot{\phi}_{1}\right)^{2}+\left(\dot{\phi}_{2}\right)^{2}\right) \\
U= & \frac{1}{2} k\left\{\left(z_{b}-z+l \phi_{b}-l \phi\right)^{2}+\left(z_{b}-z+l \phi_{b}-l \phi\right)^{2}\right\} \\
& +\frac{1}{2} k\left\{\left(z_{b}-z-l \phi_{b}+l \phi\right)^{2}+\left(z_{b}-z-l \phi_{b}+l \phi\right)^{2}\right\} \\
& +\frac{1}{2} k_{t 1}\left\{(z+s \phi)^{2}+(z-s \phi)^{2}\right\}+\frac{1}{4} k_{t 2}\left\{(z+s \phi)^{4}+(z-s \phi)^{4}\right\}
\end{aligned}
$$

Also the Rayleigh's dissipation function is obtained as follow:

$$
\begin{aligned}
F= & \frac{1}{2} C\left\{\left(\dot{z}_{b}-\dot{z}+l \dot{\phi}_{b}-l \dot{\phi}\right)^{2}+\left(\dot{z}_{b}-\dot{z}-l \dot{\phi}_{b}+l \dot{\phi}\right)^{2}\right\} \\
& +\frac{1}{2} C_{t}\left\{(\dot{z}+s \dot{\phi})^{2}+(\dot{z}-s \dot{\phi})^{2}\right\}
\end{aligned}
$$

\section{Appendix D: Dimensionless form of governing equations}

In order to generalize the conclusions of this study to a wider range of vehicles with the different parameters, the equations should be converted into dimensionless form. The process is started by definition of dimensionless time $t^{*}$ as follows:

$$
t^{*}=\frac{t}{\tau}, \quad \tau=\sqrt{(M+2 m) / k}
$$

The dimensionless linear and rotational variables are defines using rear tread $s$ and a 1 rad rotation as follows:

$$
\begin{aligned}
& z^{*}=\frac{z}{s}, z_{b}^{*}=\frac{z_{b}}{s}, \phi^{*}=\frac{\phi}{1}, \phi_{b}^{*}=\frac{\phi_{b}}{1}, \phi_{g}^{*}=\frac{\phi_{g}}{1} \\
& \phi_{p}^{*}=\frac{\phi_{p}}{1}, \phi_{c}^{*}=\frac{\phi_{c}}{1}, \phi_{1}^{*}=\frac{\phi_{1}}{1}, \phi_{2}^{*}=\frac{\phi_{2}}{1}
\end{aligned}
$$


Substituting from Eq. (2.D) into Eqs (11), (12) and (15) yields:

$$
\begin{aligned}
\ddot{z}^{*} & -\frac{2 C}{k \tau}\left(\dot{z}_{b}^{*}-\dot{z}^{*}\right)-4\left(z_{b}^{*}-z^{*}\right)+\frac{2 k_{t 1}}{k} z^{*} \\
& +\frac{k_{t 2} s^{2}}{k}\left\{\left(z^{*}+\phi^{*}\right)^{3}+\left(z^{*}-\phi^{*}\right)^{3}\right\}=Q_{z}^{*} \\
Q_{z}^{*}= & \frac{2 k_{t 1}}{k s}\left(s z^{*}-\Delta\right)+\frac{k_{t 2}}{k s}\left\{\left(s z^{*}+s \phi^{*}-\Delta\right)^{3}+\left(s z^{*}-s \phi^{*}-\Delta\right)^{3}\right\} \\
\ddot{z}_{b}^{*}+ & \frac{2 C \tau}{M_{b}}\left(\dot{z}_{b}^{*}-\dot{z}^{*}\right)+4 \frac{k \tau^{2}}{M_{b}}\left(z_{b}^{*}-z^{*}\right)=0
\end{aligned}
$$

Also the dimensionless form of Eqs (13), (14), (16) and (17) are obtained as follows:

$$
\begin{aligned}
& \ddot{\phi}^{*}-\frac{2 C l^{2} \tau}{\left(I_{x}+2 i_{x}+2 m s^{2}\right)}\left(\dot{\phi}^{*}-\dot{\phi}_{b}^{*}\right)-\frac{4 k \tau^{2} l^{2}}{\left(I_{x}+2 i_{x}+2 m s^{2}\right)}\left(\phi^{*}-\phi_{b}^{*}\right) \\
& +\frac{2 k_{t 1} s^{2} \tau^{2}}{\left(I_{x}+2 i_{x}+2 m s^{2}\right)} \phi^{*}+\frac{k_{t 2} s^{4} \tau^{2}}{\left(I_{x}+2 i_{x}+2 m s^{2}\right)}\left[\left(z^{*}+\phi^{*}\right)^{3}+\left(z^{*}-\phi^{*}\right)^{3}\right] \\
& +\frac{2 s^{2} C_{t} \tau}{\left(I_{x}+2 i_{x}+2 m s^{2}\right)} \dot{\phi}^{*}=Q_{\phi}^{*} \\
& Q_{\phi}^{*}=\frac{s \tau^{2}}{\left(I_{x}+2 i_{x}+2 m s^{2}\right)}\left\{2 s k_{t 1} \tau^{2} \phi^{*}+2 s C_{t} \dot{\phi}^{*}\right. \\
& \left.+k_{t 2} \tau^{2}\left[\left(s z^{*}+s \phi^{*}-\Delta\right)^{3}-\left(s z^{*}-s \phi^{*}-\Delta\right)^{3}\right]\right\} \\
& \ddot{\phi}_{b}^{*}+\frac{2 C l^{2} \tau}{I_{x b}}\left(\dot{\phi}^{*}-\dot{\phi}_{b}^{*}\right)+\frac{4 k \tau^{2} l^{2}}{I_{x b}}\left(\phi^{*}-\phi_{b}^{*}\right)=Q_{\phi b}^{*} \\
& Q_{\phi b}^{*}=\frac{F_{q} r_{q}}{I_{x b}} \tau^{2}
\end{aligned}
$$

The dimensionless equations related to gear train, pinion and crown wheel are presented in Eqs (7.D) to (9.D), respectively:

$$
\begin{aligned}
& \ddot{\phi}_{g}^{*}+\frac{\tau^{2}}{I_{g}} F_{e} r_{e}-\frac{\tau^{2}}{I_{g}} T_{e}=0 \\
& \ddot{\phi}_{p}^{*}+\frac{\tau^{2}}{I_{p}} F_{q} r_{q}-\frac{\tau^{2}}{I_{p}} F_{c} r_{p}=0 \\
& \ddot{\phi}_{c}^{*}+\frac{2 r_{a} \tau^{2}}{I_{c}}\left(F_{1}+F_{2}\right)-\frac{\tau^{2}}{I_{c}} F_{c} r_{c}=0
\end{aligned}
$$

Finally, tyre's dimensionless equations are as follows:

$$
\begin{aligned}
& \ddot{\phi}_{1}^{*}+\frac{\tau^{2}}{I_{1}} F_{x_{1}} R-2 \frac{\tau^{2}}{I_{1}} F_{1} r_{a}=0 \\
& \ddot{\phi}_{2}^{*}+\frac{\tau^{2}}{I_{2}} F_{x_{2}} R-2 \frac{\tau^{2}}{I_{2}} F_{2} r_{a}=0
\end{aligned}
$$

\section{References}

[1] D. Tandy, J. Neal, R. Pascarella and E. Kalis, A technical analysis of a proposed theory on tire tread belt separation-induced axle tramp, SAE Paper 2011-01-0967, 2011.

[2] H.B. Pacejka, Tire and vehicle dynamics, Elsevier, Butterworth-Heinemann, 2006.

[3] J.B. Heywood, Internal combustion engine fundamentals, McGraw-Hill, New York, 1988. 
[4] J.R. Ipser, D.A. Renfroe and A. Roberts, Solid axle tramp response near the natural frequency and its effect on vehicle longitudinal stability, SAE Paper 2008-01-0583, 2008.

[5] K.D. Kramer, W.A. Janitor and L.R. Bradley, Optimizing damping to control rear end breakaway in light trucks, SAE Paper 962225, 1996.

[6] L. Guzzella and C.H. Onder, Introduction of modeling and control of internal combustion engine systems, $2^{\text {nd }}$ ed., Verlag Berlin Heidelberg Springer, 2010.

[7] P. Falcone, G. Fiengo and L. Glielmo, Nicely nonlinear engine torque estimator, $16^{\text {th }}$ IFAC World Congress, Prague, Czech Republic, 2005.

[8] R.S. Sharp and C.J. Jones, Self-excited vibrations of truck tandem axle suspension and transmission system, Proc 7th IAVSD Symposium on Dynamics of Vehicle on Roads and Tracks, Cambridge (UK) (1981), 66-80.

[9] R.S. Sharp, The mechanics of axle tramping vibrations, Bsc, Msc, CEng, MIMechE, C137/84, 1984.

[10] R.S. Sharp, The nature and prevention of axle tramp, Proc I Mech (Aut Div) 184(3) (1969), 41-54.

[11] SAE surface vehicle recommended practice, Vehicle Dynamics Terminology, SAE J670e.

[12] T.D. Gillespie, Fundamentals of vehicle dynamics, Society of Automotive Engineers, Inc, 1992. 

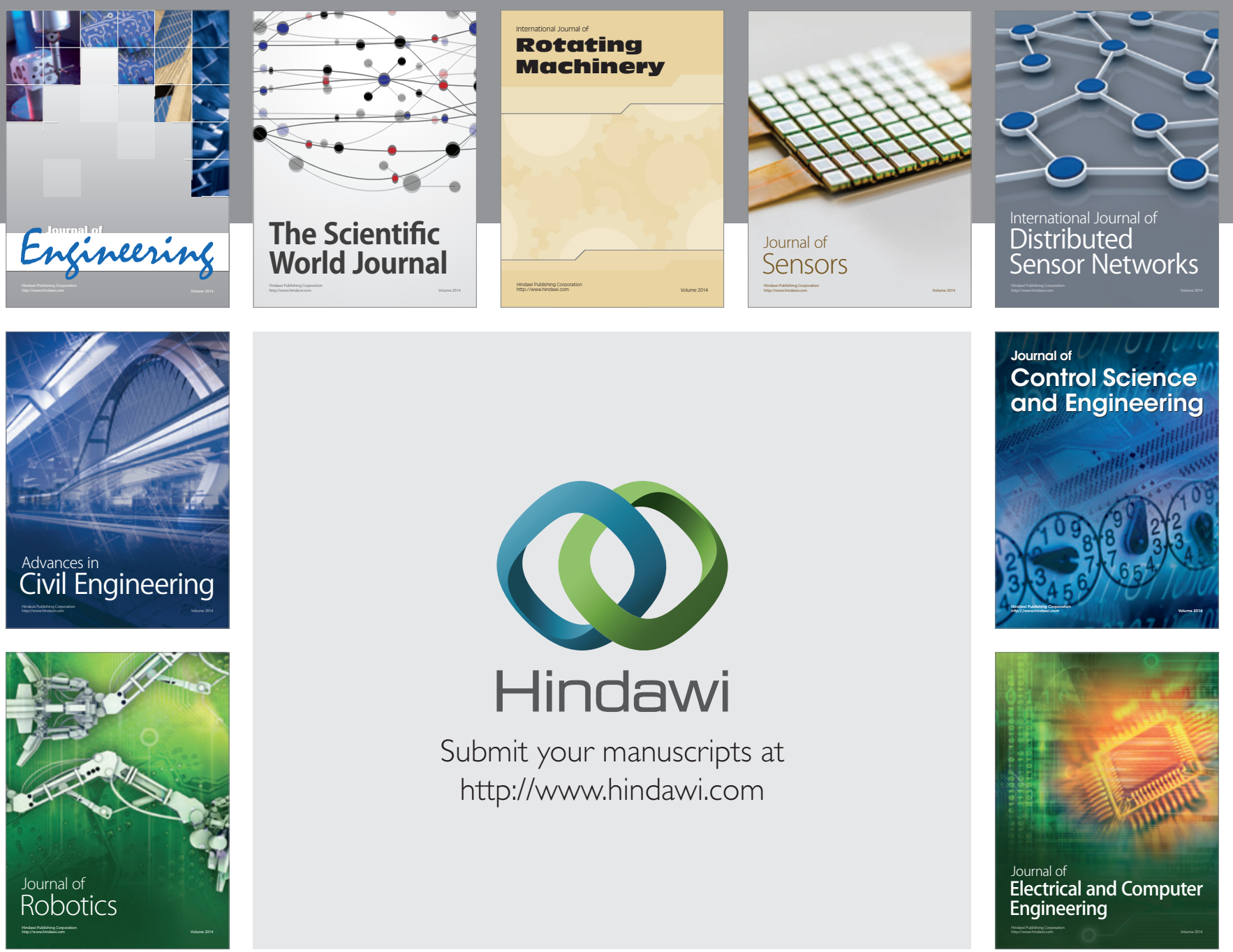

Submit your manuscripts at

http://www.hindawi.com
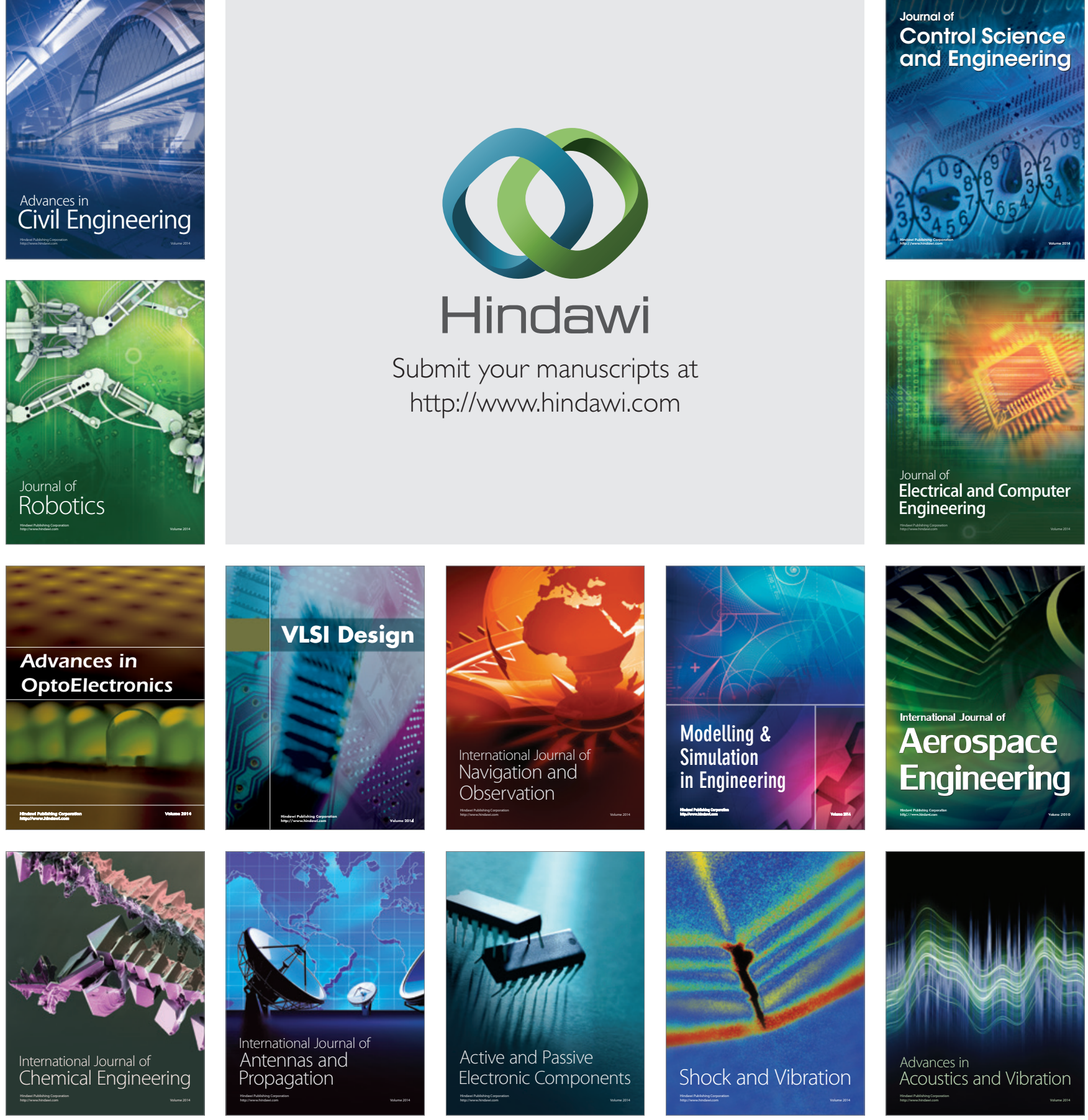